\title{
Discrete Event Switching Control Based On Hybrid System Theory Considering Lyapunov Stability
}

\author{
${ }^{1}$ D.Muralidharan, ${ }^{2}$ P.Anitha Rani, ${ }^{3}$ R.Aswani \\ ${ }^{1,}$ Assistant Professor, Department of Electrical and Electronics Engineering V.S.B Engineering College, Karur- \\ 641013, Tamilnadu, India \\ 2. PG Scholar, Power Systems, Department of Electrical and Electronics Engineering V.S.B College of \\ Engineering, Karur -641 013, Tamilnadu, India
}

\begin{abstract}
Harmonics present in the three phase converter is the major issue while it is been connected to the electric power distribution system. As the conventional model uses real time switching control, this paper deals with discrete event switching control based on hybrid system theory considering Lyapunov stability. Use of hybrid Controller is new to power electronic applications and hence the converters can provide input currents without distortion and with the unity power factor.
\end{abstract}

Keywords: Harmonics, Switching Control. Hybrid system theory, Lyapunov

\section{Introduction}

Over the last three decades, Power electronic systems represent a well-established technology that has seen significant performance improvements. In general, they involve applications of transforming electrical power from one usually unregulated form to another that is regulated to some references (e.g. considered the problem of unregulated ac to regulated dc conversion). This transformation is achieved by the use of semiconductor devices that operate as power switches, turning on and off with a high switching frequency. From the control point of view, power electronic circuits and system constitute excellent examples of hybrid systems, since the discrete switch positions are associated with different modes of continuous time dynamics.

The conventional ac/dc power converters that are connected to the line through full wave rectifiers draw a sinusoidal input ac current. Harmonic content in a current waveform flowing through the impedances in the electric utility distribution system can create harmonic voltages. These harmonics distort the local voltage waveform, potentially interfering with other electrical equipment connected to the same electrical service. Also, a distorted ac input current waveform prohibits the extraction of the maximum possible real power from the utility service. Unity power factor converters employ active wave shaping of the ac input current to ensure a sinusoidal current slope, while delivering a constant dc output voltage [1].

Typical control schemes employed in state of the art unity power factor converters have fairly low bandwidth in order to limit the impact of output voltage ripple on input power factor. In such controllers, disturbances are attended by control actions taken at most in the order of twice per line cycle [2].

The fast controller presented here executes control action at a much faster rate. As a result, the fast controller achieves a fast response time to disturbances. A key feature of our controller is its ability to reject feedback of the ripple on the dc bus capacitor by actively canceling it, so that high bandwidth control can be maintained without distorting the input ac current during steady state operation [3].

Lyapunov theory has for long time been an important tool in linear as well as nonlinear control. However, its use within the nonlinear control has been changed by the difficulties to find the Lyapunov function for a given system. If one can be found, the system is known to be

stable, but the task of finding such function has often been left to the imagination and experience of the designer [5].

The aim of this paper is the design of a control law for a three-phase boost rectifier to achieve good power and grid current control in steady state and transient operating conditions. The feedback system is globally asymptotically stable in the sense of the Lyapunov stability theory.Therefore we are interested in an extension of the Lyapunov function concept.

\section{A. Hybrid Switching Control}

\section{System Requirements And Technical Background}

The field of power electronic poses challenging control problems that cannot be treated in a complete manner using traditional modeling and controller design approaches. The main difficulty arises from the hybrid nature of these systems due to the presence of semiconductor switches that include different modes of operation 
and operate with high switching frequency. The computational power, available today, and the recent theoretical advances in the control of hybrid systems allow one to tackle these problems in a novel way that improves the performance of the system and is systematic and implemental.

The basic principle considered in power electronic circuits is the switching control. In switching control, one built bank of alternative candidate voltages depends on the configuration of switching elements in power electronic circuits. By VSI, the switching is done by a specially designed decision logic, that uses the measurements of continuous state variables, currents, to asses the performance of the candidate voltage input vector $V i$ currently in use, and also the potential performance of alternative voltage input vectors $V i-1, V i+1$. Fig. 1 shows the basic architecture employed by switching control. In this figure $\boldsymbol{u} s(\boldsymbol{V} i)$ represents the discontinuous control input voltage vector, $\boldsymbol{e}_{s}$ the grid voltage vector and $\boldsymbol{i}_{s}$ the grid input current. The dashed box is a conceptual representation of a switching controller. The top element in Fig. 1 is the selector that controls the switches, or more precisely that generates the switching control input vector $S(S 1, S 2, S 3)$. The selector is called the supervisor and its purpose is to monitor the signals that can be measured $\left(\boldsymbol{u}_{s}\left(\boldsymbol{V}_{i}\right)\right.$, current control error $\boldsymbol{i}_{s}$ ) and decide, at each instant of time, which candidate voltage vector $V i$ should be put in the feedback loop with the process. In the supervisory control, the selector combines continuous dynamic with discrete logic and is therefore a hybrid system, i.e. discontinuous input $\boldsymbol{u} s(\boldsymbol{V} i)$ and feedback continuous input current $i_{s}$.

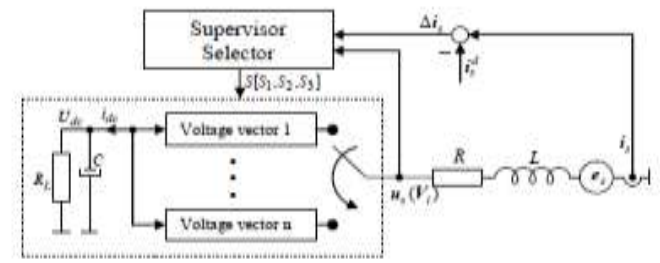

Fig. 1. Switching supervisory control

\section{B. System Configuration}

The proposed system configuration is shown in Fig. 2.The main circuit consists of a bridge rectifier made up of six power transistors with antiparallel diodes, which is connected to the three-phase supply, through an inductor $L$. A load and a capacitor $C$ are connected to the de side of the converter. The inductor $L$ performs the voltage boost operation in combination with the capacitor $C$ and at the same time acts as a low pass filter for the ac line current.

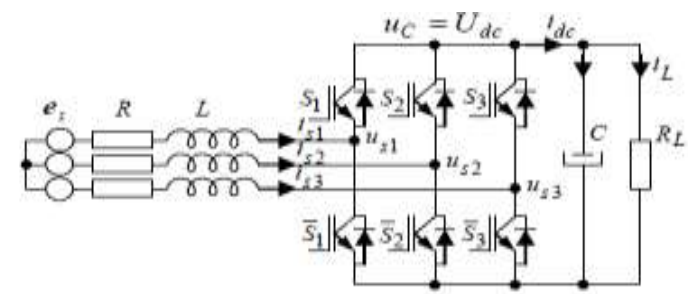

Fig. 2. System configuration

The dynamics of a three-phase boost rectifier in the three-phase reference frame for ac current control system is described as:

$$
\begin{aligned}
& L \frac{d i_{j}}{d t}+R i_{j}=e_{s j}-u_{s j}, \\
& u_{s j}=\left(d_{j}-\left(d_{1}+d_{2}+d_{3}\right) / 3\right) u_{C}
\end{aligned} \quad j=1,2,3 \quad(1)
$$

and for output voltage control system as:

$$
C \frac{d u_{C}}{d t}=i_{d c}-i_{L}=i_{1} d_{1}+i_{2} d_{2}+i_{3} d_{3}-\frac{u_{C}}{R_{L}}
$$

where $d j$ is duty ratio.

The main problem in design of the control law of the boost rectifier is occurrence of control input function in both ac current and dc voltage control systems of the converter. Both input functions depend on the same duty ratio $d j$ by different dynamics behavior of ac input and dc output of the boost rectifier. Input grid voltages and currents should be sinusoidal shaped without a phase delay between the voltage and the current and output dc voltage should be a constant value by change of voltage dependent current and parameters of the converter [7]. 
The considered control problem is the tracking of a three phase current reference signal. After defining current control error $\Delta \boldsymbol{i}_{s}=\boldsymbol{i}_{s}-\boldsymbol{i}_{s}^{\mathrm{d}}$

rewritten in error form becomes

$$
L \frac{d}{d t} \Delta i_{s}+R \Delta i_{s}=e_{s}-u_{s}\left(V_{i}\right),
$$

which collects all the disturbances (exogenous and endogenous) action on the system.

The basic principle of the current control is to manipulate the input voltage vectors so that the desired current of power electronic circuits is produced. This is achieved by choosing a converter switch combination $S_{i}$ that drives the grid current vector by directly applying the appropriate voltages $\boldsymbol{u}_{s}\left(\boldsymbol{V}_{i}\right)$. The switch positions of the three-phase converter are described using the logical variables $V \mathrm{i}$, dependent if switch $S_{\mathrm{i}}$ is ON or OFF. Each variable corresponds to one phase of the converter (Fig.2). Three-phase converter can produce $2^{3}$ voltage vector combinations; two of them are zero vectors and 6 active vectors, Fig. 3.

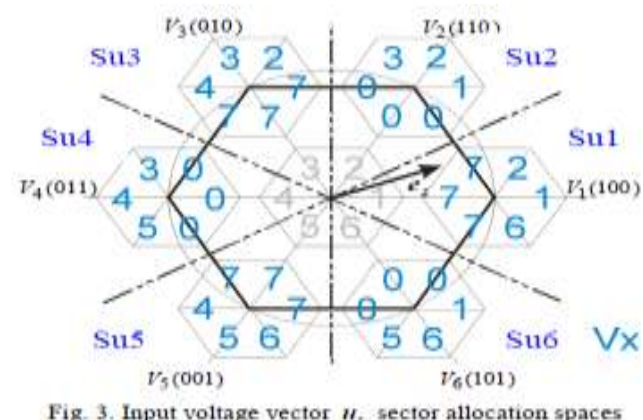

The energy flow between the input and the output side of the three-phase converter is controlled by switching matrix. By introducing the binary variables $S i$ that are "1" if particular switch $S i$ is On and "0" if switch $\mathrm{Si}$ is OFF $(\mathrm{i}=1,2,3, \ldots, 6)$ the behavior of the switching matrix can be described by the three dimensional vector $\boldsymbol{u}_{s}=U_{d c} \boldsymbol{L} \boldsymbol{S i}$, where matrix $L$ and vector $\boldsymbol{S}(S 1, S 2, S 3)$ are defined as [8]

$$
\begin{aligned}
& \mathbf{L}=\left[\begin{array}{cccccc}
1 & 0 & 0 & -1 & 0 & 0 \\
0 & 1 & 0 & 0 & -1 & 0 \\
0 & 0 & 1 & 0 & 0 & -1
\end{array}\right] \\
& \mathbf{S}^{T}=\left[\begin{array}{llllll}
S_{1} & S_{2} & S_{3} & \bar{S}_{1} & \bar{S}_{2} & \bar{S}_{3}
\end{array}\right]
\end{aligned}
$$

Relation (4) is true for the switching matrix $L$ that is depicted in Fig. 2. It essentially shows that this particular switching matrix is able to generate three independent control actions denoted as the components $S I$, $S 2$ and $S 3$ of the control vector

$$
u_{s}\left(\boldsymbol{V}_{i}\right)=U_{d c}\left[S_{1}, S_{2}, S_{3}\right]^{T} .
$$

\begin{tabular}{|c|c|c|c|c|c|c|c|}
\hline & Su1 & $\mathrm{Su} 2$ & $\mathrm{Su} 3$ & Su4 & Sus & Su6 \\
\hline & & 100 & 110 & 010 & 011 & 001 & 101 \\
\hline Sdio & 000 & V7 & vo & V7 & Vo & V7 & V0 \\
\hline Sdi1 & 100 & V1 & V1 & V7 & Vo & V7 & V1 \\
\hline Sdi2 & 110 & $\mathrm{~V} 2$ & V2 & $v_{2}$ & Vo & V7 & Vo \\
\hline Sdi 3 & 010 & V7 & V3 & $\sqrt{3}$ & $\mathrm{~V}_{3}$ & V7 & Vo \\
\hline Sdi4 & 011 & V7 & Vo & V4 & V4 & $\mathrm{V} 4$ & V0 \\
\hline Sdi5 & 001 & V7 & V0 & V7 & V5 & V5 & V5 \\
\hline Sdi6 & 101 & V6 & Vo & V7 & V0 & V6 & V6 \\
\hline Sdi7 & 111 & V7 & vo & V7 & V0 & V7 & V0 \\
\hline
\end{tabular}

The components, i.e. switch position of the converter, are generated by a look up table (Table I and Fig. 3).

Table I - Look-up Table

\section{Discrete-event Current Control}

To control the current $\boldsymbol{i} s$, the sector of the grid voltage $\boldsymbol{e}_{s}$ is recognized first. Based on the known sector, the input voltage vector $\boldsymbol{u}_{s}\left(\boldsymbol{V}_{i}\right)$ (the transistor switching pattern) for the current control is selected in respect to the current control error, related to Lyapunov stability condition. To consider a space vector representation of the grid voltage $\boldsymbol{e}_{s}$, the voltage is represented as vector that rotates around the origin. Six active switching vectors of the three-phase transistor converter result in six active input voltage vectors denoted $V_{1} \ldots V_{6} ; V_{0}$ and 
$V_{7}$ are two zero voltage vectors. According to signs of the phase voltages $u s 1$, us 2 and $u s 3$,(Fig 1) the phase plane is divided into six sectors denoted Su1 ... Su6, Fig. 3. In regards to the situation, the grid voltage space vector $\boldsymbol{e}_{s}$ is in sector 1. In this sector, voltage vectors $\boldsymbol{V}_{0}, \boldsymbol{V}_{1}, \boldsymbol{V}_{2}, \boldsymbol{V}_{6}$ and $\boldsymbol{V}_{7}$ are selected for the current control. $\boldsymbol{V}_{0}, \boldsymbol{V}_{7}$ are two zero vectors, while $\boldsymbol{V} 1, \boldsymbol{V}_{2}, \boldsymbol{V}_{6}$ are three nearest adjacent live input voltage vectors to this sector. With the use of the discrete event system theory, five input voltage vectors $\boldsymbol{V}_{0}, \boldsymbol{V}_{1}, \boldsymbol{V}_{2}, \boldsymbol{V}_{6}$ and $\boldsymbol{V}_{7}$ are recognized as discrete states of the system. Events represent the allowed transition among the discrete states i.e. allowed switching.

Discrete States: $\mathrm{V}_{0}, \mathrm{~V}_{1}, \mathrm{~V}_{2} \ldots \ldots . \mathrm{V}_{7}$

Events : Allowed Switching

Transition :Between the different Voltage Vector

\section{Lyapunov-based Control Strategy}

The analysis of the three-phase boost rectifier is a very challenging theoretical problem because we add to the complexity, of controlling a nonlinear multivariable uncertain system, the fact that the control signal lives in finite set $S\left(S_{1}, S_{2}, S_{3}, \sim S_{1}, \sim S_{2}, \sim S_{3}\right)$, hence we are confronted with the hybrid system.

On the basis of Lyapunov stability theory, a positive definite scalar function $V$, as a candidate for the Lyapunov function, is to be found, such that the total energy of the system is continuously dissipated. In such a case, any nonlinear system must eventually settle down

to an equilibrium point. For the Lyapunov function candidate

$$
V=(1 / 2) \Delta i_{s}{ }^{T} \Delta i_{s}=(1 / 2)\left(i_{s}-i_{s}^{d}\right)^{T}\left(i_{s}-i_{s}^{d}\right),
$$

the stability requirement will be fulfilled if control can be selected as such, that the derivative of the Lyapunov function candidate is negative $\mathrm{V}=\Delta \mathrm{i}_{\mathrm{s}}^{\mathrm{T}} \Delta \mathrm{i}_{\mathrm{s}} \leq 0$. Derivatives of the current control error (6) may be expressed with the voltage equation

$$
(d / d t)\left(i_{s}-i_{s}^{d}\right)=(1 / L)\left(e_{s}-R i_{s}-u_{s}\left(V_{i}\right)\right)-(d / d t) i_{s}^{d},
$$

where $\boldsymbol{i}_{s}{ }^{d}, \boldsymbol{i}_{s}$ are desired and actual grid current,

$\boldsymbol{u}_{s}\left(\boldsymbol{V}_{i}\right)$ is voltage control input, $R \boldsymbol{i}_{s}$ is resistive voltage drop and $\boldsymbol{e}_{s}$ is ac input voltage.For $d(\Delta i s) / d t=0$ the equivalent control voltage [11] can be expressed as:

$$
u_{\text {equ }}=e_{s}-R_{i}-L(d / d t) i_{s}{ }^{d}
$$

and derivative of Lyapunov function is:

$$
\bar{V}=\left(\mathbf{i}-\mathbf{i}_{\mathrm{s}} \mathbf{d}\right)\left(\mathbf{u}_{\text {equ }}-\mathbf{u}(\mathbf{V})\right) / \mathbf{L}<\mathbf{0}
$$

The conditions for the sequential switching of the power converter are selected as:

$$
\begin{aligned}
& S_{1}=(1 / 2)(1-\operatorname{sign}(A)), S_{2}=(1 / 2)(1-\operatorname{sign}(B)), \\
& S_{3}=(1 / 2)(1-\operatorname{sign}(C))
\end{aligned}
$$

Where

$$
\begin{aligned}
& A=\left(i_{s a}-i_{s a}^{d}\right) \\
& B=-(1 / 2)\left(i_{s a}-i_{s a}^{d}\right)-(\sqrt{3} / 2)\left(i_{s b}-i_{s b}^{d}\right), \\
& C=-(1 / 2)\left(i_{s a}-i_{s a}^{d}\right)+(\sqrt{3} / 2)\left(i_{s b}-i_{s b}^{d}\right)
\end{aligned}
$$

which is evolved from the Lyapunov function derivative. When $U d c$ has enough magnitude that $\dot{V} \leq 0$, than $V \rightarrow 0$ and $i{ }^{d} \rightarrow i s . S 1, S 2$ and $S 3$ represent the switching state of the three-phase power converter. Notice that if $S 1, S 2, S 3$ equal to zero simultaneously, no current is delivered to the dc load.

The proposed logical event-driven grid current control can be realized in the form described in Table I, where states of grid current control error are presented by $\operatorname{sign}(D j)(D j=S 1, S 2, S 3)$ and currently active voltage sector is presented by sign Es (eS1,eS2,eS3). To further

improve the presentation, active voltage vectors are marked in Table I with a blue background. Because the transition between converter switch states is performed by switching only one converter leg, converter switching frequency grid current chattering are reduced.

\section{E. Discretized Output Voltage Control}

The discretized dc output voltage control will be designed for the control system expressed by (2). The unity power factor of ac/dc boost rectifier will be achieved for $u C=u d, u q=0$ in d-q output reference frame.

The output voltage control error 


$$
\sigma_{\mathrm{u}}=\mathrm{u}_{\mathrm{c}}{ }^{\mathrm{d}}-\mathrm{u}_{\mathrm{c}}
$$

determines the input dc current $i d c$ which also plays the role of the rectifier output current. With $\dot{\sigma}_{u}=-d_{u} \sigma_{u}$ we get a robust PI-control algorithm [8]:

$$
i_{d c}(k)=i_{d c}(k-1)+\frac{G_{u} C}{T}\left(\left(1+T d_{u}\right) \sigma_{u}(k)-\sigma_{u}(k-1)\right) .
$$

In the proposed algorithm the output load resistance $R L$ does not appear, therefore the control is independent of its value. The resistive load can change its value in transient states, i.e. which includes step change of its value. The rectifier dc current $i d c$, which forms an input for dc boost rectifier part is influenced by a dc output voltage error $\sigma u(k)$, dc bus capacitor $C$, used sampling time $T$ and feedback gain $G u$. The asymptotical stability of algorithm is guaranteed with the proper choice of parameter $d u$. The reference input active power would be determined with dc current $i d c$ and bus voltage $U d c$ :

$$
\mathrm{p}^{\mathrm{d}}=\mathrm{U}_{\mathrm{dc}} \mathrm{i}_{\mathrm{dc}}
$$

In Fig. 5, the complete block diagram of proposed discretized output voltage and ac input current control scheme in hybrid switching control is presented.

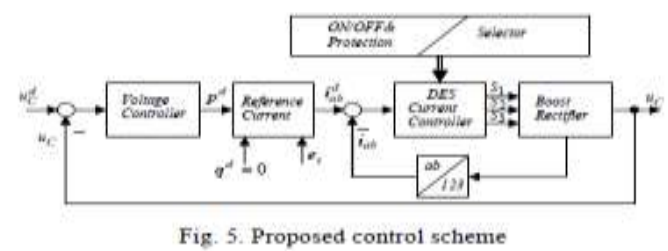

\section{Unity Power Factor}

In the proposed nonlinear control scheme, which has ability to obtain sinusoidal shaped ac input current, we shall profit from the benefits of robust switching control. These benefits are insensitivity to parameter variations and excellent decoupling properties. The sinusoidal form of ac currents is assured with the proposed discrete event current control. The actual current tracks the desired value without delay, it is robust to the variations of resistance $R$ and inductance $L$ and it decouples the influences between supply phases. We assume that the supply frequency remains constant. The time constant that determines the transient of ac input currents $T s=L / R$ is some order smaller than the bulky designed dc time constant $T d c=R L C$ which determines the slow change of output dc voltage $u C$. This physical property of time dependent variability of ac input current and slow change of output dc voltage can be profitably used to express the relation between the input ac power and dissipated output dc power.

The sinusoidal form of ac currents is assured with the proposed discrete event current control:

$$
I_{s}=I_{s 0} e^{j \omega_{0} t+\varphi}
$$

where $\omega 0=314,159 \mathrm{~s}-1$ is mains frequency of ac input voltages. Input ac power is expressed with dot product of:

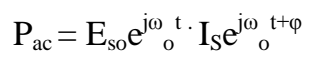

The boost rectifier will take only real power component from the ac input with phase delay between ac voltage and ac current $\phi \rightarrow 0$, i.e. this means unity power factor. Output dc power will be less for losses in boost rectifier and is expressed with ac signals

$$
\mathrm{P}_{\mathrm{ac}}=3 \mathrm{I}_{\mathrm{s}} \mathrm{E}_{\mathrm{s}}
$$

and dc output power

$$
\mathrm{P}_{\mathrm{dc}}=\mathrm{I}_{\mathrm{L}} \mathrm{U}_{\mathrm{C}}
$$

which will be completely converted to heating of load resistor $R L$. By neglecting of switching and conducting losses of converter the relation $P a c \approx P d c$ holds. This means that sinusoidal shaped input current must be modulated in current control plant align with supply voltage $\boldsymbol{e} s$ and than the unity power factor is reached. 


\section{Implementation}

\subsection{STEPS TO IMPLEMENT DISCRETE EVENT MODEL}

- Obtaining feedback signals such as input ac current, input ac voltage, output dc current, output dc voltage to generate current error signals

- Generating input voltage sector states

- Implementing the table obtained for current error signals and voltage sector states using Hybrid controller

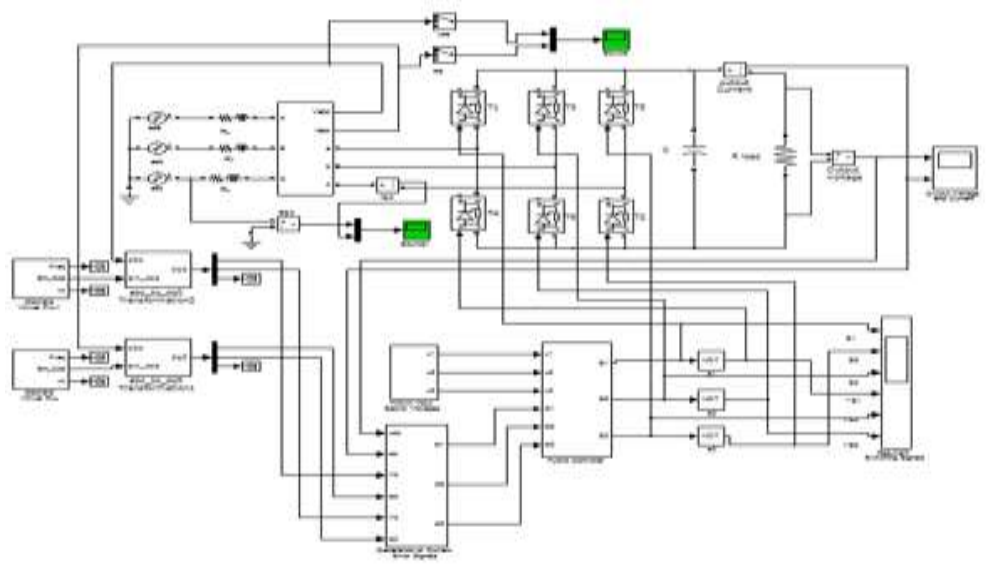

Figure 5.5: Simulink model of proposed method

The simulink model shown in Figure 5.5 has three voltage sources phase shifted by $120^{\circ}$ in order to obtain the three phase voltage input to the converter. The resistance and inductance are connected serially to the three phases of the voltage source. The values of the resistances and inductances are the same in each phase.Three phase voltage and current measurement is used to measure the current and voltage signals. Three phase voltage and current in abc axis is transformed to dqo axis using abc to dqo transformation block in simulink. The o axis signal is blocked using the terminator. The available voltage and current signals in dq axis is given as the feedback signals to generate current error.

Three phase converter is composed of six thyristor switches, where three switches are connected to the top of the three phase leg and other three to the bottom of the leg. The gate pulses to the switches are given from the output of the hybrid controller. As no two switches in the same leg can be switched on at the same instant, the switching pulses to the upper and lower switches in a leg are inverted. The resistive load is connected at the output side of the three phase converter. The filter capacitor is connected in parallel to the resistive load to remove the noise in the circuit. The output voltage and current are then measured using the voltage and current measurement simulink blocks. The harmonics is analysed for three phase input current after developing the discrete event model using FFT analysis.

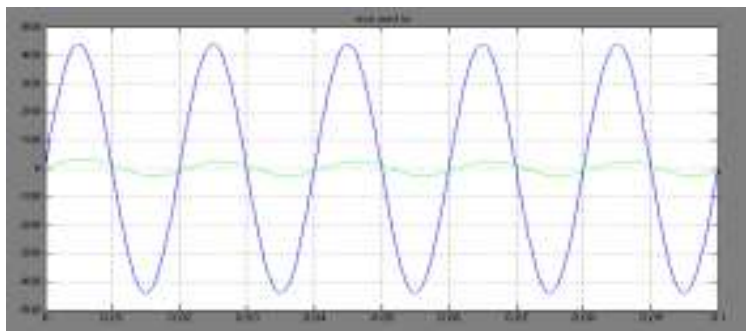

Figure 5.9(a) Input ac voltage and current in Phase a

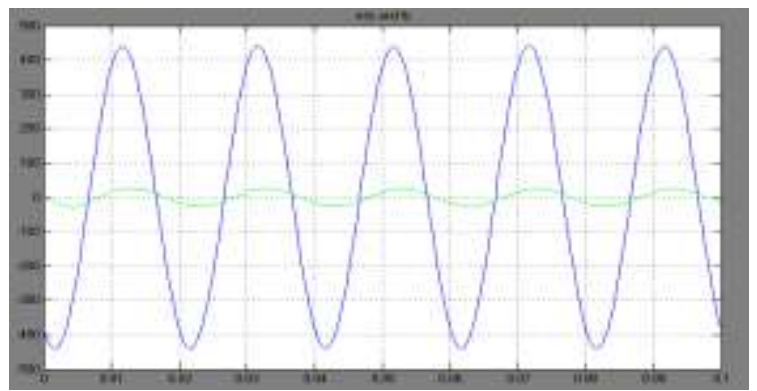

Figure 5.9(b) Input ac voltage and current in Phase b 


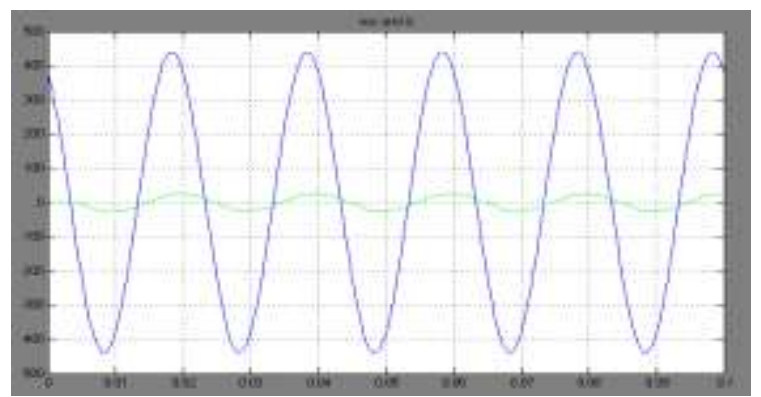

Figure 5.9(c) Input ac voltage and current in Phase c

The ac current in the three phases of the converter after implementing the discrete event model is analyzed for harmonics and shown in Figure 5.10.

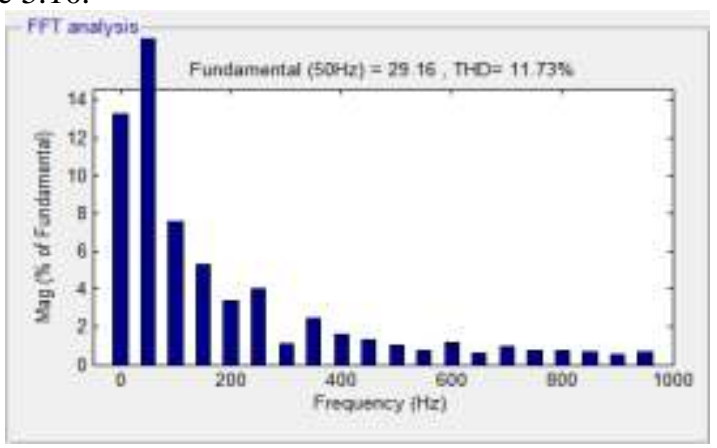

\section{Conclusion}

The hybrid based discrete event model is designed for auxiliary steering and protection functions for a three phase rectifier.The proposed discrete event model generates switching signals and controls the switches of the three phase converter. The power factor is improved by neglecting the reactive power and controlling only the active power. The stability is taken into account for analyzing the voltage and current using Lyapunov method. The feedback system is globally asymptotically stable in the sense of Lyapunov.

The performance of the three phase converter for the proposed event driven current control and SVPWM method of control were compared by simulation. The performance parameters considered here are power factor and Total Harmonic Distortion (THD). The input ac current harmonics is reduced by $5.18 \%$ and the power factor is considerably improved

\section{References}

[1] H.M. Suryawanshi, M.R. Ramteke, K.L. Thakre and V.B. Borghate, "Unity-Power-Factor Operation of Three-Phase AC-DC Soft Switched Converter based on Boost Active Clamp Topoly in Modular Approach," IEEE Tran. Power Electronics, vol. 23, no. 1, pp. 229-236, 2008

[2] J. Dannehl and F.W. Fuchs, "Discrete Sliding Mode Current Control of Three-phase Grid-Connected PWM Converters," in Proc. 13th European Conference on Power Electronics and Applications, EPE'09, pages 1-10, 2009.

[3] A. Polic and K. Jezernik, "Closed-loop matrix based model of discrete event systems for machine logic control design," IEEE Trans. Industrial Informatics, vol. 1, no. 1, pp. 39-46, 2005

[4] P. Antoniewicz and M.P.Kazmierkowski, "Virtual-Flux-Based Predictive Direct Power Control of AC/DC Converters with Online Inductance Estimation," IEEE Tran. Ind. Electronics, vol. 55, no. 12, pp. 4381 - 4390, 2008.

[5] A. Sabanovic, K. Jezernik and N. Sabanovic, "Sliding Modes Applications in Power Electronics and Electrical Drives," in Lecture notes in control and information sciences, 1st ed., Y.U. Xinghuo, X.U. Jian-Xin, (Ed.). Berlin: Springer, pp. $223-251,2002$.

[6] A. Tilli A and A. Tonielli, "Sequential Design of Hysteresis Current Controller for Three-Phase Inverter," IEEE Trans. Ind. Electronics, vol. 45, no. 5, pp. 771-781, 1998.

[7] P.J.G. Ramadge and W.M. Wonham, "The Control of Discrete Event Systems," Proceedings of the IEEE, vol.77, no. 1, pp. 81- 99, 1989.

[8] L.E. Holloway, B.H. Krogh and A. Giua, "A Survey of Petri Net Methods for Controlled Discrete Event Systems," Journal of Discrete Event Dynamical Systems: Theory and Applications, vol. 7, no. 2, pp. 151-190, 1997.

[9] V.I. Utkin, Sliding Modes in Control and Optimization. Berlin: Springer Verlag, 1992.

[10] E. Monmasson and M.N. Cirstea, "FPGA Design Methodology for Industrial Control Systems - A Review," IEEE Tran. Ind. Electronics, vol. 54, no. 4, pp. 1824-1842, 2007.

[11] D. Hercog, M. Čurkovič, G. Edelbaher and E. Urlep, "Programming of the DSP2 board with the Matlab/Simulink," in Proc. of IEEE ICIT 2003, Maribor, Slovenia, pp. 709-713, 2003

[12] B. Bomar: Implementation of a micro programmed control in FPGA. IEEE Trans. Ind. Electronics, vol. 49, no. 2, pp. 415-422, Apr. 2002 . 